\title{
Identifying Gender from Facial Parts Using Support Vector Machine Classifier
}

\author{
Sayatani Ghosh \\ Department of Computer Science and Engineering \\ University of Calcutta \\ Kolkata, India
}

\author{
Samir Kumar Bandyopadhyay \\ Department of Computer Science and Engineering \\ University of Calcutta \\ Kolkata, India
}

\begin{abstract}
Gender classification can be stated as inferring female or male from a collection of facial images. There exist different methods for gender classification, such as gait, iris, hand shape and hair, it is probably better way to find out gender based on facial features. In this paper SVM basic kernel function has been employed firstly to detect and classify the human gender Image into two labels i.e. (1) male and (2) female. The gender classifier achieves over $96 \%$ accuracy.
\end{abstract}

Keywords: Machine Learning; Support Vector Machine; Kernel; Cross Validation; Histogram Equalization.

\section{INTRODUCTION}

Machine Learning can be divided into (1) Supervised Learning in which case the target output is explicitly specified and (2) Unsupervised Learning where the training data comprises of a set of input vectors with no corresponding target values.

Support Vector Machine is one such Supervised Learning Model which has its own learning algorithms to analyses data and perform data classification/regression while eluding the over fitting issue. [1] SVM with its variety of similarity functions termed as Kernel Functions read inputs and predict the similarity between them. A kernel function is performed in the input space over a high dimensional feature space.

A linearly separable problem can be effortlessly classified into distinct groups by a separating hyper plane. Nevertheless, the role of a Kernel Function is realized evidently when the SVM classifier is implemented on inseparable data. For non- linear data, the kernel functions are used to non- linearly map the input data to a highdimensional space. The new mapping can then be linearly separated [2].

Thus the principle idea behind our endeavor is to appraise the performance of SVM basic kernel functions which has been employed to detect and classify the human gender into (1) male and (2) female. These functions read as input the feature(s) of human facial image(s). The proposed algorithm is then executed on the elementary features of a human facial image i.e. eyes, nose, lips and their all possible combinations. Finally based on the accuracy percentage of the computed result the admissible outcome of the Kernel Functions has been realized.

\section{Kernel Overview}

The primitive Kernel functions of the Support Vector Machine Classifier are (1) Linear Kernel. (2) Gaussian-RBF Kernel. (3) Polynomial Kernel. (4) Sigmoid Kernel. Each kernel is associated with parameter(s) with its own default values [3-4]. These parameters are appropriately tuned to evaluate the performance of the kernel function in predicting the accuracy percentage of the tested results. It leads to the generation of a hyper plane which maximizes the margin and better generalization is achieved. Consequently, the points nearest to the hyper plane is assigned positive weight within the higher-dimensional feature space. These points are referred to as support vectors.

\section{LITERATURE REVIEW}

In the year 1992, Boser, Guyon, and Vapnik in COLT-92 first acquainted Support Vector Machine as a set of interrelated supervised learning techniques incorporated into a family of generalized linear classifiers which can be applied to a data set for classification and regression [5-7], The classifier can be applied on variety of data set including text, image, audio, video to achieve a desired level of classification. One such renowned application of SVM Classifier is in the field of Human Gender Detection and its Classification. The connotation of Gender Recognition was first identified in the field of research and development at the inception of 1990s. Similarly, Golomb et al. [8] used multi layer neural network to recreate a solution to the gender classification issue. Nearly 900 manually aligned facial images were compressed into 40 images on which the classification was performed. An error rate of 8.1 was 
reported. The research work was based on human facial features as the primary components on which the gender classification algorithm was implemented.

Shobeirinejad and Gao [8] also proposed an Interlaced Derivative Pattern (IDP) to extract facial features. The IDP image is a four-channel derivative image representation technique where images are viewed from different angles which includes $0^{\circ}, 45^{\circ}, 90^{\circ}$, and $135^{\circ}$. This method too emphasizes on the extraction of distinct facial features and convey crucial information about gender identification. Similar task was also accomplished by LU et al. [9-10]. In their experiment CAS-PEAL database was used. $480 \times 360$ Grey scale images were transformed to normalized whole facial image and normalized internal facial image. Experiments were performed based on a technique on seven facial regions of varying resolution. In order to improve the overall performance this method performed fusion of multiple facial regions. With regard to all the previous work, in the year 2015, an allied task of Human Gender Classification was attained by Mrs. Sayantani Ghosh \&Prof. Samir Kumar Bandyopadhyay. They used the 'lip' as the primary feature of the frontal facial image. Utilizing the aforesaid extracted feature, a similar experiment was percolated using multi class SVM to consummate the task of Age Detection and its classification into predefined class labels i.e. 'child', 'adult' and 'old'. [11] In this paper, analogous to the preceding methodologies, a refined technique has been proffered. The dataset chosen for the examination of the proposed algorithm comprises of 100 JPEG frontal facial images which includes 50 males and 50 female images. The Region of Interest(ROI) principle is employed for the identification of the location of the feature(s) like eyes, nose, lips. The specified algorithm is then implemented on the dissociated feature(s) and their variety of combinations i.e. eye, nose lip, eyes nose, nose lip, eyes lip eyes, nose lip. An evaluation process computed the accuracy percentage of the resultant outcome for each input feature(s). The primitive objective of this procedure is to estimate the performance of the primary kernel functions i.e. Linear, Gaussian, and Polynomial kernel.

\section{PROPOSED METHODOLOGY}

This section emphasizes on the analysis of the proposed technique being adapted to attain the task of Gender Detection and its Classification using variety of combinations of human facial features. In addition, a metamorphic reasoning on the performance of the Kernel Functions in predicting the accuracy percentage of activated result has been accomplished.

\section{Algorithm}

Step 1: Input JPEG Image Set.

Step 2: Metamorphose individual image to grey scale version.
Step 3: Percolate histogram equalization on each grey scale image.

Step 4: Extract the primary features from the facial image. Step 4.1: for each extracted feature image perform a sequential execution of the below specified steps from $2 \mathrm{D}-1 \mathrm{D}$ Step 4.1.1: Reshape the extracted image

Step 4.1.2: Generate a feature vector for each extracted feature image(s).

Step 4.1.3: Associate with each image i.e. for each row vector a class label. Assign +1 to female image and -1 to male image.

end

Step 5: Shuffle each row of Feature Vector.

Step 6: Cross- Validate the updated matrix and generate the train set and the test set.

Step 7: Select appropriate kernel with its parameter value(s) of the SVM classifier and train the appropriate known data set.

Step 8: Apply test method of SVM to test the unknown data set.

Step 9: Retrieve the final classified result.

Step 10: End.

\section{Implementation}

The implementation details of the above stated methodology are enlisted as follows:

- Software used- MATLAB routine of the libSVM 3.18 toolbox. [12]

- $\quad$ Input Set- An image set comprising $100 \mathrm{Jpeg}$ frontal facial images (50 males and 50 females). Each image resized to dimension $128 * 115$.

- Individual image pre-processed to remove unwanted components, enhance the image and improve the image contrast.

- The train set is executed to generate the train vector using the svmtrain () routine.[12]

- The class labels of the unknown data set are determined by invoking the svmclassify () routine. [12]

The extraction principle applied on facial images to extract its distinguishable features is the Region of Interest (ROI) principle. Some preprocessing steps are taken into account for the extraction in a better way. The steps followed in this principle are matriculated as follows.

- Select an appropriate window dimension based on the location of the facial feature of the human frontal facial image. 
- The feature is then identified and extracted from the selected location using the imcrop () function.

- Each extracted image is resized to a one dimensional vector.

- Feature Vectors of the extracted feature/ feature pair i.e 'eyes', 'nose', 'lips', 'eyes nose', 'nose lips', 'eyes lips' are generated. With each row vector of the Feature Matrix class label of +1 is assigned for the female image and a class label -1 is assigned for the male image.

The diagrammatic Representation of the Feature Extraction Phase is illustrated in the following figures.

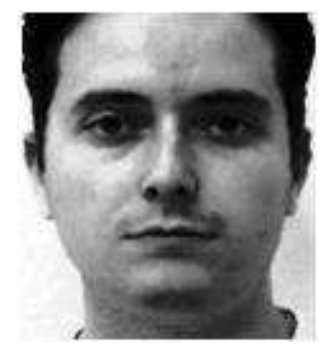

Fig 1: Original Gray Scale Image

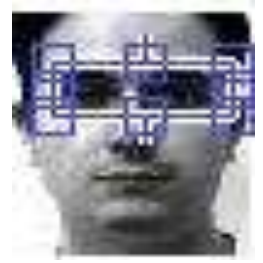

Fig 2: Unveiled Region of Interest 1

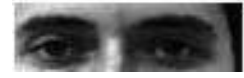

Fig 2.1: Extracted Eyes Region

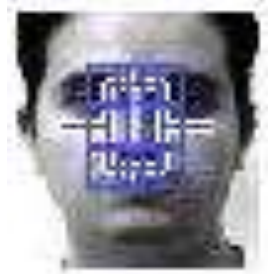

Fig 3: Unveiled Region of Interest 2

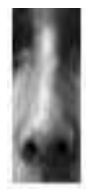

Fig 3.1: Extracted Nose Region

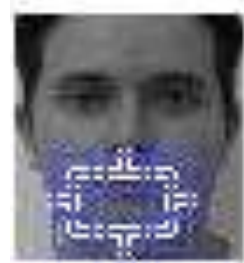

Fig 4: Unveiled Region of Interest3

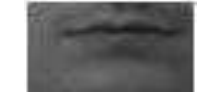

Fig 4.1: Extracted Lip Region

In shuffling phase the individual row vector of the feature matrix is shuffled. The shuffled matrix is then subjected to the next level of processing i.e. cross validation. The main objective of shuffling is to obtain a cross validated result with improved precision. The following tables describes different phases involved for finding gender.

Table 1: Tabular Representation of the class labels of the original feature vector consisting of 100 chosen images where ' 1 ' represent the female gender and ' 0 ' represent the male gender.

\begin{tabular}{|l|l|l|l|l|l|l|l|l|l|l|l|l|}
\hline 1 & 1 & 1 & 1 & 1 & 1 & 1 & 1 & 1 & 1 & 1 & 1 & 1 \\
\hline 1 & 1 & 1 & 1 & 1 & 1 & 1 & 1 & 1 & 1 & 1 & 1 & 1 \\
\hline 1 & 1 & 1 & 1 & 1 & 1 & 1 & 1 & 1 & 1 & 1 & 1 & 1 \\
\hline 1 & 1 & 1 & 1 & 1 & 1 & 1 & 1 & 1 & 1 & 1 & 0 & 0 \\
\hline 0 & 0 & 0 & 0 & 0 & 0 & 0 & 0 & 0 & 0 & 0 & 0 & 0 \\
\hline 0 & 0 & 0 & 0 & 0 & 0 & 0 & 0 & 0 & 0 & 0 & 0 & 0 \\
\hline 0 & 0 & 0 & 0 & 0 & 0 & 0 & 0 & 0 & 0 & 0 & 0 & 0 \\
\hline 0 & 0 & 0 & 0 & 0 & 0 & 0 & 0 & 0 & & & & \\
\hline
\end{tabular}

Table 2: Tabular Representation of the Shuffled Matrix.

\begin{tabular}{|l|l|l|l|l|l|l|l|l|l|l|l|l|}
\hline 0 & 0 & 0 & 1 & 1 & 0 & 1 & 0 & 0 & 0 & 1 & 0 & 1 \\
\hline 1 & 0 & 0 & 1 & 1 & 1 & 0 & 0 & 1 & 1 & 1 & 0 & 1 \\
\hline 0 & 1 & 0 & 0 & 1 & 0 & 1 & 1 & 1 & 1 & 1 & 0 & 0 \\
\hline 1 & 1 & 1 & 1 & 1 & 0 & 1 & 1 & 0 & 1 & 1 & 1 & 0 \\
\hline 1 & 0 & 0 & 0 & 0 & 1 & 0 & 0 & 1 & 1 & 1 & 0 & 1 \\
\hline 1 & 1 & 0 & 0 & 0 & 0 & 0 & 1 & 1 & 0 & 1 & 1 & 0 \\
\hline 1 & 0 & 0 & 1 & 0 & 0 & 0 & 0 & 0 & 1 & 1 & 1 & 0 \\
\hline 1 & 0 & 0 & 0 & 0 & 0 & 0 & 1 & 1 & & & & \\
\hline
\end{tabular}


Cross Validation of the Shuffled Matrix is one of the key steps in Gender Recognition Algorithm. It resolves the issues like over fitting of images. Besides if the original data set is appropriately cross validated, it can be effortlessly divided into the train set and the test set. The size of the train set and the test set however depends on the degree of the cross validation technique. Like the hold out technique divides the original set into two equal sized sets, while the other techniques like ' 10 fold' cross validation and ' 5 fold 'cross validation dissociates the primitive data into 10 segments and 5 segments respectively. Each time one segment is tested to predict the class labels of the undetermined set after acquiring the result of the training of the remaining (n-1) segments.

Table 3: Tabular Representation of '10 fold 'Cross Validated Data Set. Here the train set comprises of the data with class label ' 1 'which includes 90 instances while the test set comprises of the data with class labels '0'which includes 10 instances of the 100 prime data set.

\begin{tabular}{|l|l|l|l|l|l|l|l|l|l|l|l|l|}
\hline 1 & 1 & 0 & 1 & 1 & 1 & 1 & 1 & 1 & 1 & 1 & 1 & 1 \\
\hline 1 & 1 & 1 & 0 & 1 & 1 & 1 & 1 & 1 & 1 & 1 & 1 & 1 \\
\hline 1 & 1 & 1 & 1 & 1 & 1 & 1 & 1 & 1 & 1 & 1 & 1 & 1 \\
\hline 1 & 1 & 1 & 1 & 1 & 1 & 1 & 1 & 1 & 1 & 1 & 0 & 0 \\
\hline 1 & 1 & 1 & 0 & 1 & 1 & 1 & 1 & 1 & 1 & 0 & 1 & 1 \\
\hline 0 & 1 & 1 & 1 & 1 & 1 & 1 & 1 & 1 & 1 & 1 & 1 & 1 \\
\hline 1 & 1 & 1 & 1 & 1 & 1 & 1 & 1 & 1 & 0 & 1 & 1 & 0 \\
\hline 1 & 0 & 1 & 1 & 1 & 1 & 1 & 1 & 1 & & & & \\
\hline
\end{tabular}

The cross validated data are then trained using the svmtrain () function of the libSVM toolbox. [12] Finally, the trained result is subjected to the svmclassify () routine of the libSVM toolbox [12] which classifies the test set and predicts the class labels of the unknown set.

Table 4: Tabular Representation of the output class labels of the Tested Result

\begin{tabular}{|l|l|l|l|l|l|l|l|l|l|}
\hline 0 & 1 & 1 & 0 & 0 & 1 & 1 & 1 & 0 & 0 \\
\hline
\end{tabular}

Table 5: Tabular Representation of the actual class labels of the original Feature Vector

\begin{tabular}{|l|l|l|l|l|l|l|l|l|l|}
\hline 0 & 1 & 1 & 0 & 0 & 1 & 1 & 1 & 0 & 0 \\
\hline
\end{tabular}

\section{EXPERIMENTAL RESULT}

The program code of the above stated algorithm has been written using MATLAB version R2013a. On proper excerption of the key kernels available in SVM i.e. (1) Linear (2) RBF and (3) Polynomial. The graphical Analysis of the performance of different Kernels are presented in the following figures.

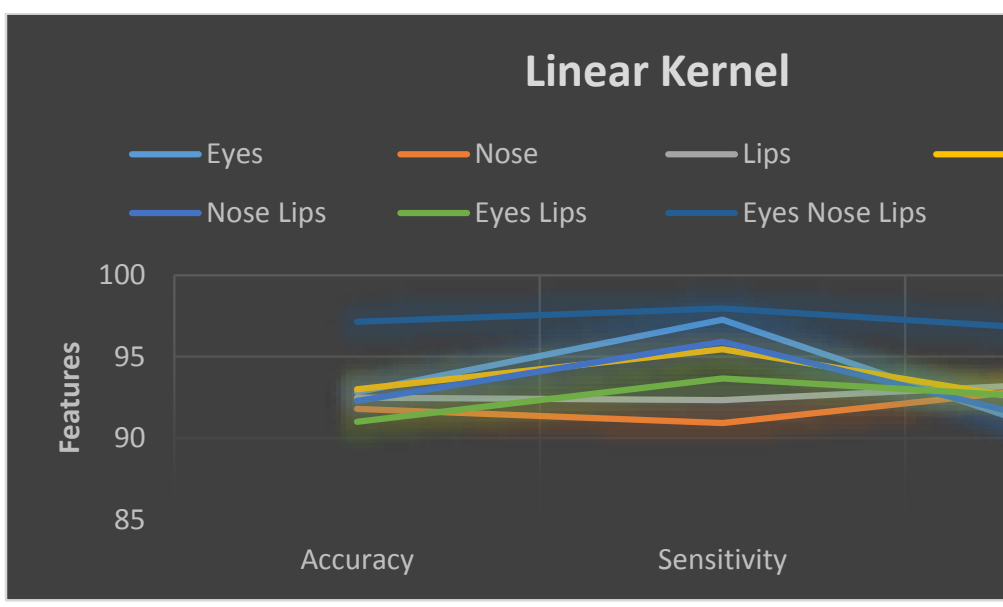

Fig 5: Graphical Analysis of the performance of Linear Kernel

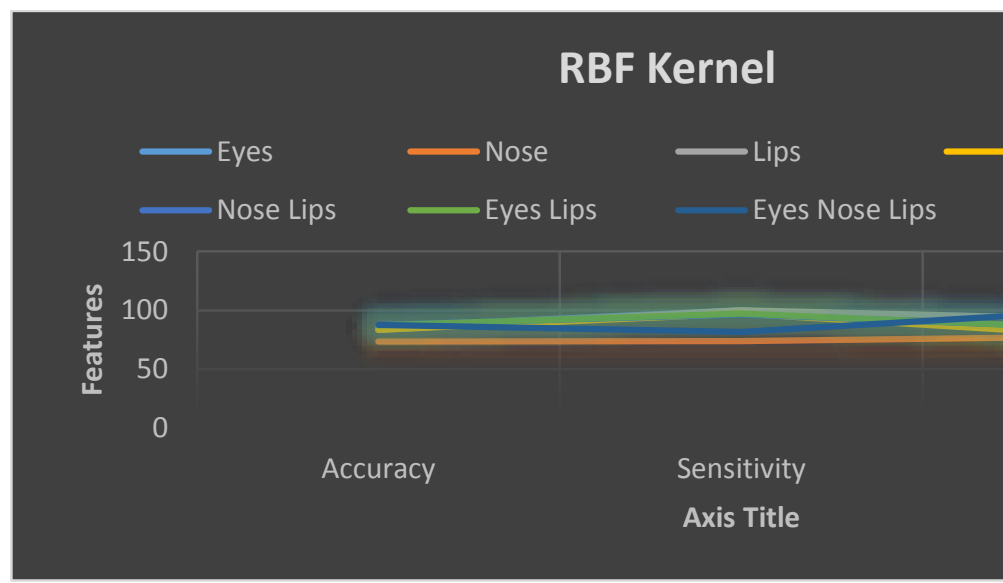

Fig 6: Graphical Analysis of the performance of RBF Kernel 


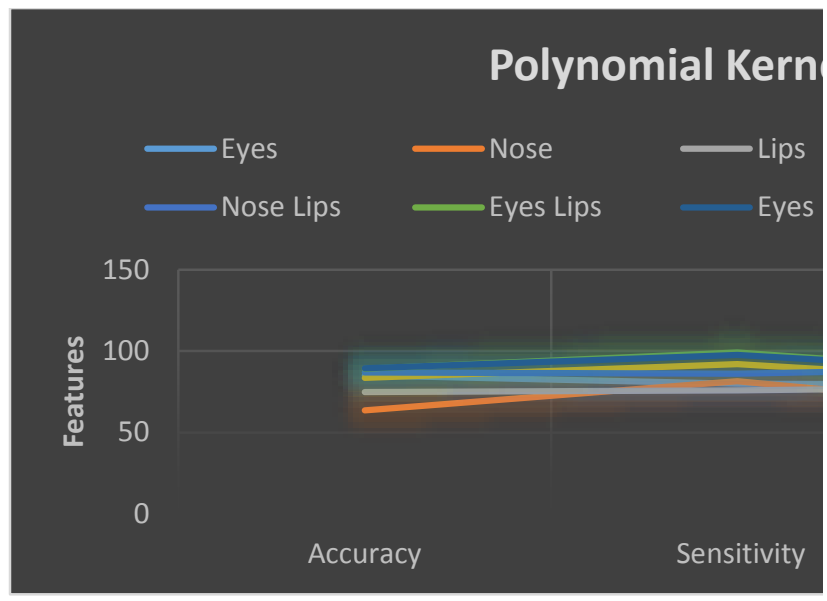

Fig 7: Graphical Analysis of the performance of Polynomial Kernel

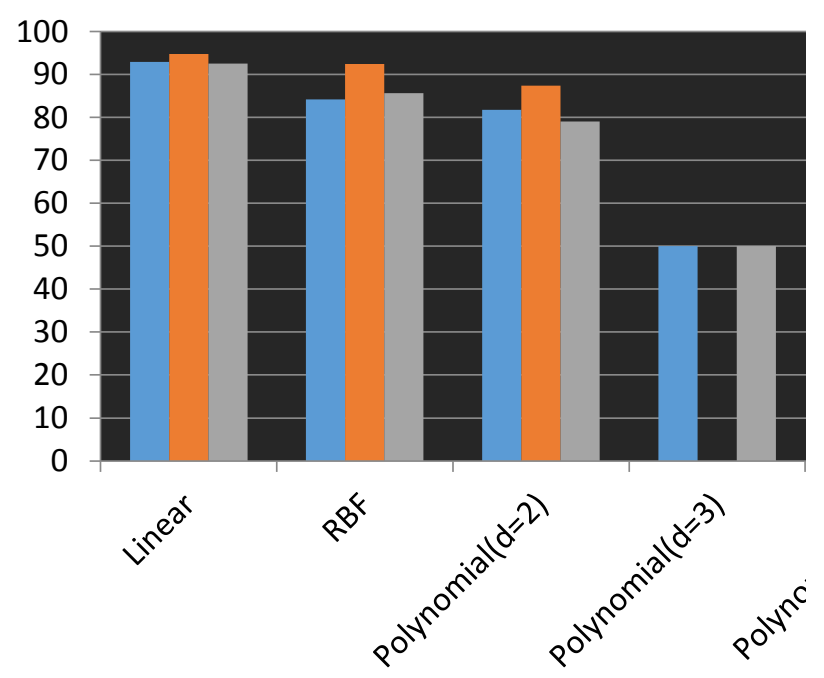

Fig 8: Graphical Representation of Comparative Analysis of Kernels Functions

\section{CONCLUSION}

The test results as stated in the previous sections characterize the performance of the kernel functions in the evaluation of Gender Classification based on the combination of features of Human Facial Image. With this paper we have endeavored to prolongate our previous task of Human Gender Classification. [11] In our experiment, the test results verified the behavior of a kernel on a given data set. The Linear Kernel yielded the best result when implemented on data set comprising of 100 Images linearly separated into 50 males and 50 female images compared to
RBF and Polynomial Kernel. The Polynomial Kernel which is best suited for non-linear data set generated poor result for degree' $s=3$ ' or ' $s=4$ '. Thus its degree was lowered to ' $d=2$ ' that led to the generation of favorable yet no so high result. However, the RBF kernel did not make a notable performance since firstly, the data set is inherently linearly separated and secondly the feature vector includes an increased feature count over input instances. Thus with an idea of achieving upgraded performance of the proposed methodology, the data set shall be increased with more instances.

\section{REFERENCES}

[1] Vikramaditya Jakkula, "Tutorial on Support Vector Machine (SVM)".

[2] Martin Hofmann, "Support Vector Machines - Kernels and the Kernel Trick", June 26, 2006.

[3] B. Yekkehkhany1, et.al. "A Comparison Study of Different Kernel Functions for SVM-based Classification of Multi-Temporal Polarimetry SAR Data", The International Archives of the Photogrammetry, Remote Sensing and Spatial Information Sciences, Volume XL-2/W3, 2014. pp.281-285.

[4] Chih-Wei Hsu, et.al. "A Practical Guide to Support Vector Classification", Initial version: 2003, Updation: May 19, 2016.

[5] Rita McCue, "A Comparison of the Accuracy of Support Vector Machine and Naive Bayes Algorithms in Spam Classification", University of California at Santa Cruz Nov 29, 2009.

[6] Hsuan-Tien Lin, et.al. "A Study on Sigmoid Kernels for SVM and the Training of non-PSD Kernels by SMO-type Methods", Department of Computer Science and Information Engineering National Taiwan University.

[7] Bernhard E Boser, EECS Department University of California Berkeley, et.al. "A Training Algorithm for Optimal Margin Classifiers".

[8] B.A. Golomb, et.al. "Sexnet: A Neural Network identifies Sex from human faces," Advance in neural information processing systems, pp. 572-577, 1990.

[9] Ameneh Shobeirinejad, et.al. "Gender classification using interlaced derivative patterns.", In Proc. IEEE International Conference on Pattern Recognition. 2010; 1509-1512.

[10] Li LU, et.al. "Gender classification of facial images based on multiple facial regions." World Congress on Computer Science and Information Engineering. 2009;4852.

[11] Sayantani Ghosh, et.al. "Gender Classification and Age Detection Based on Human Facial Features Using MultiClass SVM", British Journal of Applied Science \& Technology 10(4): 1-15, 2015, Article no. BJAST.19284 ISSN: 2231-0843.

[12] Chih-Chung Chang, et.al. " LIBSVM -- A Library for Support Vector Machines”, Version 3.21 - December 14, 2015. 